\title{
A Fast, Lightweight, and Reliable File System for Wireless Sensor Networks
}

\author{
Biswajit Mazumder ${ }^{*}$, Jason O. Hallstrom ${ }^{\dagger}$ \\ * School of Computing, Clemson University, bmazumd@clemson.edu \\ ${ }^{\dagger}$ Institute for Sensing and Embedded Network Systems Engineering, Florida Atlantic University, jhallstrom@fau.edu
}

\begin{abstract}
Sensor nodes are increasingly used in critical applications. A file storage system that is fast, lightweight, and reliable across device failures is important to safeguard the data that these devices record. A fast and lightweight file system should enable sensed data to be sampled and stored quickly for later transmission, while imposing a small resource footprint. A reliable file system should provide storage integrity in the face of hardware, software, and other failures.

We present the design and implementation of LoggerFS, a fast, lightweight, and reliable file system for wireless sensor networks which uses a hybrid memory design consisting of RAM, FRAM, and flash. LoggerFS is engineered to provide fast data storage, have a small memory footprint, and provide data reliability across system failures. Additionally, LoggerFS is designed to be power efficient. LoggerFS adapts a log-structured file system approach, augmented with data persistence and reliability guarantees. A caching mechanism allows for flash wear-leveling and fast data buffering. We present a performance evaluation of LoggerFS using a prototypical in situ sensing platform, and demonstrate between $50 \%$ and $800 \%$ improvements for various workloads using the FRAM write-back cache.
\end{abstract}

\section{INTRODUCTION}

Typical WSN deployments consist of resource-constrained sensor nodes tasked with monitoring local conditions. Applications such as disaster management [5], structural monitoring [19], and volcanic activity monitoring [30] require constant recording, transmission, and processing of sensed data. Some applications require immediate transmission [5], while others require periodic transmission. The ability to store data in situ is essential in both scenarios [6]. Applications with immediate transmission requirements may have periods of radio connectivity loss, during which they may need to buffer sensed data. Other applications may employ buffering with periodic transmissions to extend battery life.

Permission to make digital or hard copies of all or part of this work for personal or classroom use is granted without fee provided that copies are not made or distributed for profit or commercial advantage and that copies bear this notice and the full citation on the first page. Copyrights for components of this work owned by others than ACM must be honored. Abstracting with credit is permitted. To copy otherwise, or republish, to post on servers or to redistribute to lists, requires prior specific permission and/or a fee. Request permissions from Permissions@acm.org. EMSOFT'16, October 01-07, 2016, Pittsburgh, PA, USA (c) 2016 ACM. ISBN 978-1-4503-44852/16/10? \$15.00 DOI: http://dx.doi.org/10.1145/2968478.2968486
Storing buffered data in RAM is not always feasible as the size of primary memory in typical sensor nodes is often small [9]. For example, the Atmel ATMega644P microcontroller (MCU), used in our custom sensing platform, has only $4 \mathrm{~KB}$ of RAM [2]. The maximum supported RAM size in a Texas Instruments MSP430 MCU, used in the Telos platform [22], is $64 \mathrm{~KB}$ [14]. While flash memory is slower than RAM, it offers a viable alternative due to its larger memory size. However, some sensor applications have the capability to acquire data at continuously high rates, overwhelming flash memory, which offers comparatively slow write-erase speeds. Flash memory is also characterized by a limited number of write-erase cycles, potentially "wearing out" due to frequent writes. These limitations have prompted efforts to develop improved storage technologies. Non-Volatile Static RAM (NVSRAM), Ferroelectric RAM (FRAM), Ovonic Unified Memory (OUM), and Magnetoresistive RAM (MRAM) are potential successors of flash technology - all boasting performance advantages, including low power consumption, a large number of write-erase cycles, and fast read and write performance. Exploiting the advantages of these newer technologies is the key to designing fast, lightweight, and reliable file systems.

Memory technologies that combine the read and write speeds of RAM with the non-volatility of flash can be used to design fast and reliable file systems. In the past, traditional EEPROM technologies have been used to achieve file system reliability in embedded systems [6]. EEPROM differs from flash in that it can be reprogrammed one byte at a time. However, EEPROM is slower than flash, which supports page-based writes and block-based erases; EEPROM is not suitable for applications with fast data update rates.

Table 1 presents a comparison of memory technologies, available or under development. For a memory technology to be used in a resource-constrained environment, the memory device should have low active and stand-by current consumption. To support a fast file system, the device should provide fast read and write access times. Finally, the memory technology should support a large number of read/write-erase cycles to avoid wearing out. While SRAM and DRAM allow fast read and write access (84ns and $10 \mathrm{~ns}$, respectively), they are volatile in nature, i.e., they lose their data when power is removed. Some older NVSRAM devices achieve non-volatility using battery backed SRAM [8], while newer devices use non-volatile elements (EEPROM cells) [24]. NVSRAM devices typically have data access speeds comparable to SRAM devices (20ns). How- 
Table 1: Memory Technology Comparison

\begin{tabular}{|c|c|c|c|c|c|c|c|}
\hline & $\begin{array}{c}\text { DRAM } \\
(\mathrm{MD} 51 \mathrm{~V} 65165 \mathrm{E})\end{array}$ & $\begin{array}{c}\text { SRAM } \\
(71 \mathrm{~V} 016 \mathrm{SA} 10)\end{array}$ & $\begin{array}{c}\text { NVSRAM } \\
\text { (CY14B104NA) }\end{array}$ & OUM & $\begin{array}{c}\text { MRAM } \\
(\mathrm{MR} 2 \mathrm{~A} 16 \mathrm{~A})\end{array}$ & $\begin{array}{c}\text { FLASH } \\
(\text { SST39LF010) }\end{array}$ & $\begin{array}{c}\text { FRAM } \\
(\text { FM22L16) }\end{array}$ \\
\hline Non Volatile & No & No & Yes & Yes & Yes & Yes & Yes \\
\hline Non Destructive Read & No & Partial & Yes & Yes & Yes & Yes & No \\
\hline Direct Overwrite & Yes & Yes & Yes & Yes & Yes & No & Yes \\
\hline Write Cycles & $\infty$ & $\infty$ & $2 * 10^{6}$ & $10^{12}$ & $\infty$ & $10^{6}$ & $>10^{14}$ \\
\hline Read Cycles & $\infty$ & $\infty$ & $\infty$ & $\infty$ & $\infty$ & $\infty$ & $>10^{14}$ \\
\hline Write Speed & $84 n s$ & $10 n s$ & $20 n s$ & $10 n s$ & $35 n s$ & $200 \mu s$ & $110 n s$ \\
\hline Read Speed & $84 n s$ & $10 n s$ & $20 n s$ & $20 n s$ & $35 n s$ & $45 \mathrm{~ns}$ & $110 \mathrm{~ns}$ \\
\hline Erase Speed & $84 n s$ & $10 n s$ & $20 n s$ & $50 n s$ & $35 n s$ & $18 \mathrm{~ms} /$ sector & $110 n s$ \\
\hline Active Current & $120 \mathrm{~mA}$ & $65 m A$ & $65 m A$ & $\mathrm{n} / \mathrm{a}$ & $105 m A(\mathrm{AC})$ & $5 m A$ & $8 m A$ \\
\hline Stand-by Current & $2 m A$ & $10 \mathrm{~mA}$ & $5 m A$ & $\mathrm{n} / \mathrm{a}$ & $18 m A(\mathrm{AC})$ & $1 \mu A$ & $5 \mu A$ \\
\hline
\end{tabular}

ever, NVSRAM allows limited write cycles (like flash) and exhibits high active and stand-by current, making it unsuitable for many sensing applications. In comparison, FRAM offers a high number of read and write cycles $\left(10^{14}\right)$ [25], along with fast data access (110ns for reads, writes, and erases). FRAM is characterized by low active and stand-by current consumption ( $8 m A$ and $5 \mu A$, respectively). Unlike flash, FRAM also allows direct byte overwrites.

We present the design of LoggerFS - a fast, lightweight, and reliable file system for embedded network systems. The implementation uses a hybrid memory design consisting of RAM, FRAM, and flash. LoggerFS provides fast sensor data access, while imposing only a small main memory footprint. LoggerFS adapts a log-structured file system design, supplemented with a lightweight consistent data structure update mechanism to provide file system reliability in the presence of faults. LoggerFS is also power-efficient. The design includes a novel FRAM-based caching mechanism which supports flash wear-leveling and fast data buffering during periods of high-rate sensing. We present a performance evaluation on a prototypical in situ sensing platform.

\section{RELATED WORK}

In this section, we survey some of the most well-known file systems for embedded systems, many of which use logstructured design principles [23]. The design of LoggerFS is influenced in part by JFFS [31], designed by Woodhouse et al. Prior to JFFS, the traditional design approach was to use a block device interface to interact with flash memory. JFFS was among the first to introduce a log-structured design specifically for flash devices. Versions have been implemented for the 2.x Linux kernels; those support garbage collection and checksum-based fault detection. However, JFFS lacks a fault-tolerance mechanism, including the ability to recover from system crashes. Additionally, although JFFS was designed for use in embedded devices, its implementation does not consider resource-constrained systems.

Dai et al. present ELF [6], which also implements a logstructured file system for embedded devices. ELF uses a group of pages in a linked-list to represent files in memory. It offers a rich set of features which provide support for random file overwrites and hierarchical directory structures. ELF also allows files to grow in memory after they have been created, handling flash fragmentation via a garbage collector. Interestingly, ELF stores files in NAND flash, and filesystem metadata, such as directory structures, in EEPROM to limit the wear on flash. It also supports limited crash recovery for certain special files. Crash recovery metadata is also stored in EEPROM. Our design differs from ELF in that we do not allow files to grow once they have been created. LoggerFS also does not support directory structures and random file overwrites since embedded sensor file systems are mostly used for logging sensed data. Additionally, the amnesiac nature of LoggerFS (old records are discarded when new data arrives to a full file) allows it to operate without a garbage collector. This makes LoggerFS much faster and lighter-weight, while providing complete file system crash recovery capabilities.

Doh et al. present a file system which uses NVRAM to store file metadata and flash to store file content [7]. They present a model to predict the amount of NVRAM required for a specific flash size to achieve the optimal usable flashto-NVRAM ratio for peak performance. For realistic file access workloads, they achieve maximum performance improvements of $600 \%$, and an average improvements of $437 \%$ over YAFFS [4], another flash-based file system, successfully demonstrating that flash-based file systems can use NVRAM to accelerate performance. LoggerFS shares similarities with this file system in that it also employs non-volatile memory (FRAM) to accelerate data access. However, unlike this file system, LoggerFS provides complete crash recovery capabilities. Yoon et al. [32] and Kim et al. [18] also present hierarchical storage architectures, introducing FRAM for metadata storage to accelerate reads and writes to flash. Again, these architectures do not provide crash recovery capabilities and do not target resource-constrained platforms.

Tsiftes et al. present the Coffee file system [29] for flashbased devices, implemented for the Contiki operating system [10]. Coffee uses a linked-list of page structures to represent files in memory and flash, and uses micro-logs to handle file modifications. This allows Coffee to use fixed-size file metadata in RAM. Coffee uses a first-fit algorithm for page allocation in flash, and files are stored as a contiguous list of pages. If files grow larger than their current allocated size, they are moved to a different location in flash. Coffee implements a garbage collector that provides some wear-leveling guarantees. Because of the complex, processcentric implementation of the Coffee file system (requiring significant processing power), LoggerFS outperforms Coffee in terms of read and write performance. Coffee also provides limited crash recovery, making it possible to lose entire file contents during a system failure e.g., due to a loss of power. This lack of data persistence makes Coffee unsuitable for many critical applications.

Gay et al. present the Matchbox file system [12] as part of the TinyOS operating system [20], with design goals similar 
to LoggerFS. Matchbox provides support for data reliability - specifically, the ability to detect corruptions and limit data loss to files being edited during system failure without corrupting file metadata. Matchbox also implements atomic metadata updates, which allows it to support atomic read and write operations. Memory allocation in flash is done using a free page bitmap. The Matchbox design aligns with many of our design choices: wireless sensor file systems typically do not need support for security, hierarchical file system structures, random file access, or multiple file opens. LoggerFS supports atomic read and write operations via a novel consistent data structure approach, in addition to providing full crash recovery capabilities. Unlike LoggerFS, Matchbox lacks a complete crash recovery solution such that it is able to reinitialize itself after a system failure. Matchbox also requires certain NOR flash capabilities which limit its usability. Additionally, the RAM and ROM footprints for Matchbox are high, increasing linearly with the number and size of files in the system [29]. In comparison, the RAM footprint of LoggerFS is constant for files of any size.

Gal et al. present TFFS [11], a transactional file system for resource-constrained embedded devices. TFFS implements an efficient, pruned, versioned search tree for file representation in memory; fast search and file access; and atomic operations. Transactions and atomic file operations are stored as part of a log before being committed to memory. For small flash devices, TFFS uses a small amount of primary memory to provide a mapping from real to logical sector numbers. However, for larger flash devices, this memory requirement is much larger, which makes TFFS unsuitable for embedded devices with limited RAM.

Cao et al. present a hierarchical file system and an associated shell interface as part of the LiteOS operating system [3]. LiteOS provides a Unix-like file system and operating abstraction for wireless sensor networks, with complete directory structures and user-shell interaction. The unit of storage in the file system is the block. LiteOS stores file metadata (control blocks) in EEPROM, and data (storage blocks) in flash. An in-memory bit vector provides information about the used blocks in EEPROM and flash. Interestingly, LiteOS also implements a search-by-name feature, which is able to handle string queries. While LiteOS is feature-rich and can be manipulated using shell commands and application development libraries, it is memory-intensive. Furthermore, LiteOS does not guarantee reliability; consistency may be compromised across system failures.

There has been significant work on storage systems for flash-based embedded devices [3, 6, 7, 11, 12, 20, 29,31]. However, none of these systems are designed to sustain bursty, high data rates, while allowing complete crash recovery and data persistence in the presence of device failures. Moreover, LoggerFS is unique in its lightweight design and its use of FRAM as a metadata store and write-back cache to support fast reads and writes.

\section{DESIGN CONSIDERATIONS}

Designing a file system for sensor systems poses unique challenges due to the scarcity of computational resources afforded by most hardware platforms. The small size of primary memory (RAM) and limited battery power require a reevaluation of common file system design choices, which tend to be resource-intensive.

\subsection{Batch Data Transmissions}

WSNs typically consist of resource-constrained sensor devices that sense and transmit data to one or more base stations, where resource-intensive computations are performed. Since data transmission is energy-intensive, a common strategy is to store data locally (in RAM), and to later send batched data. With the advent of inexpensive flash memory technology, sensor nodes equipped with flash devices are able to store large amounts of sensed data. There are, however, challenges associated with flash storage. The write granularity of flash memory is one page, ranging from 512B to $8 \mathrm{~KB}$, while the erase granularity is a block, ranging from 64 to 128 pages. The amount of data (in pages) that can be written to flash is limited by the size of RAM, since the data must first be buffered.

\subsection{Data Sampling Rates}

Mission-critical sensor applications can produce continuous data at high rates. This can overwhelm flash memory devices, which write at slower speeds than the incoming data rate due to slow write-erase cycles. The devices also support writes and erases at larger page and block granularities, respectively, compared to the data sample sizes. Flash memory, which is characterized by limited write-erase cycles, can also "wear out" due to frequent data updates during highrate sensing. Batching data writes in RAM can help with wear-leveling to a limited extent and can sustain short bursts of high-rate sampling. However, for systems with continuously high sampling rates, this is not a feasible solution. Instead, some form of non-volatile memory which is larger in size than the available RAM may be used as cache.

\subsection{Data Persistence}

It is important for sensor devices deployed as part of mission-critical applications to include a robust file system so that data can be accessed reliably, even across device failures (e.g., power disruptions, operating system crashes, etc.) Designing such a file system usually requires checkpointing/snapshotting [27]. However, even the simplest checkpointing/snapshotting solutions are resource-intensive and not suited for implementation on sensor nodes.

\subsection{Data Considerations}

To design a fast file system for sensor nodes, it is important to understand the types of data stored on these nodes, as well as the associated access patterns. WSNs primarily deal with three types of data:

- Sensor Data: All recorded data is generated by the sensors attached to a node. In many applications, this data must be batched in RAM or a persistent storage device before it is transmitted to a base station. Some sensors have the ability to generate data very quickly. The sampled data is typically recorded in a sequential manner. During transmission, data is usually read back in a FIFO manner, transmitted, and finally deleted from the storage device.

- Binary Program Image Data: The ability to reprogram wireless sensor nodes in large installations is important. Some network reprogramming strategies involve transferring an entire binary program image to the sensor nodes $[13,17,28]$. Some of the more sophisticated, incremental approaches transfer only a diff 
between the new and old images, which is then used to reconstruct the new binary image on each node $[15$, 16,21]. Regardless of the reprogramming strategy, the transferred data must be stored in a reliable manner. Errors in binary program image data can cause sensor nodes to stop functioning.

- Configuration Data: Configuration data contains the parameter values necessary to configure the operation of sensor nodes (e.g., sampling frequency, file size, transmission rate, etc.) Integrity of this data is important since faulty values can cause sensor nodes to malfunction or stop working altogether. However, configuration data is updated infrequently. Configuration data is usually stored in EEPROM, in the absence of other persistent storage devices; reads and writes to EEPROM are usually energy-intensive and slow.

The number of files that must be supported by a file system for sensor nodes is limited, as a substantial percentage of the associated operations are typically for storage and retrieval of sensed data. The number of files required in such a system is on the order of the number of available sensors. Configuration data requires limited additional memory, while the space required to store a copy of a binary program image cannot be greater than the size of the device's programmable flash memory.

\section{FILE SYSTEM DESIGN}

LoggerFS is optimized to address the design considerations discussed in Section 3, including the identified data and file access patterns. Figure 1 illustrates the hardware architecture of a sensor node running LoggerFS. The sensor node's MCU includes integrated RAM and EEPROM, both limited in size. We extend these facilities with an external FRAM module and an external flash module.

Data generated by attached sensors $\left\{\mathrm{S}_{1}, \ldots, \mathrm{S}_{\mathrm{n}}\right\}$ is processed by the MCU and ultimately written to flash. The data is later read from flash and transmitted to a base station using a wireless module. Alternatively, binary program image data (or the image diff) is received by the wireless module from the base station and stored in flash memory. The data is later read from flash, and the program image is reconstructed and written to the programmable flash memory of the sensor node $[15,16,21]$. Configuration data is stored as a special file in external flash.

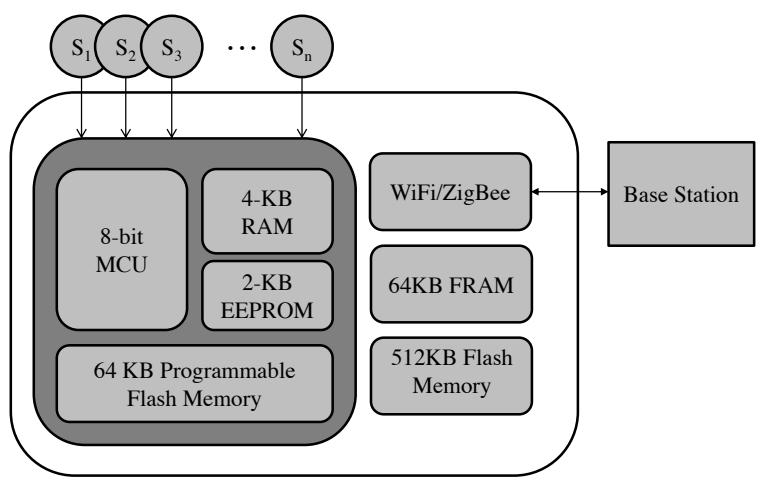

Figure 1: LoggerFS Hardware Architecture
Figure 2 summarizes the content structure across the three memory modules in the hybrid design. Files are stored in flash memory. LoggerFS uses a flat structure since the total number of files in an embedded system is expected to be low. The structure supports fast lookup and update mechanisms.

During execution, each file is represented in primary memory (RAM) by a metadata record. Updates to a file require updates to the metadata, both in volatile and non-volatile memory. As discussed in Sections 3.1 and 3.2, there are challenges associated with writing data to flash memory. Smaller updates, such as file metadata, tend to be more problematic than large block updates, due to the page-sized write granularity of flash memory. Consequently, LoggerFS stores file metadata in FRAM. Since FRAM allows for fast, byte-sized write operations, frequent updates to the metadata do not pose a challenge.

FRAM storage is partitioned into two parts, a metadata store and a write-back cache. In addition to storing the file metadata, the metadata store maintains the data structures required to support the buffer cache, which are loaded in primary memory during execution. The write-back cache is used to facilitate fast write caching in FRAM during periods of high-rate sensing. The size of the write-back cache is significantly larger than the free space available in RAM, thus allowing for efficient write-batching and flash wear-leveling.

The runtime file metadata (associated with files being accessed), the variables required to maintain the file metadata (e.g. stack variables and pointers), and cache data structures are stored in RAM. To prevent the loss of file system state due to the loss of runtime variables stored in RAM (e.g., as a result of unexpected power loss), LoggerFS uses a lightweight consistent data structure to achieve data persistence. We discuss the data structure in Section 4.3.

\subsection{Files in Flash}

Figure 3 illustrates the logical view of file and record layouts in flash. Sensor nodes are primarily used to log sensed data, and to transmit this data to a base station. New files are usually not created once a system has been deployed. For these reasons, LoggerFS supports a fixed number of files, configured during system installation. Each attached sensor is paired with a file using a unique identifier; the maximum number of files supported depends on the size of external flash memory and the configuration of file sizes. File sizes are configured at installation time based on the sensing rate and maximum required critical data period. Sensor data logged during a single sampling period is called a record.

Each file in LoggerFS is a circular buffer capable of containing variable-sized records. The update granularity is one record. New records are appended to the end of a file; old records are always read and/or deleted from the beginning. If a file is full, and the node is unable to communicate with the base station, new records overwrite old records. This

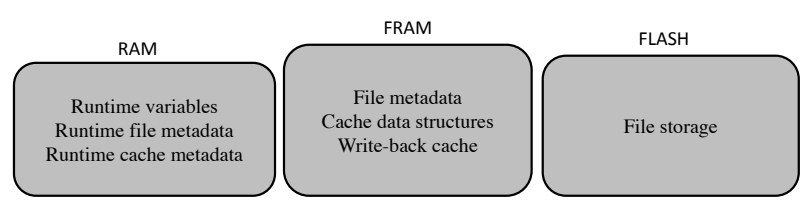




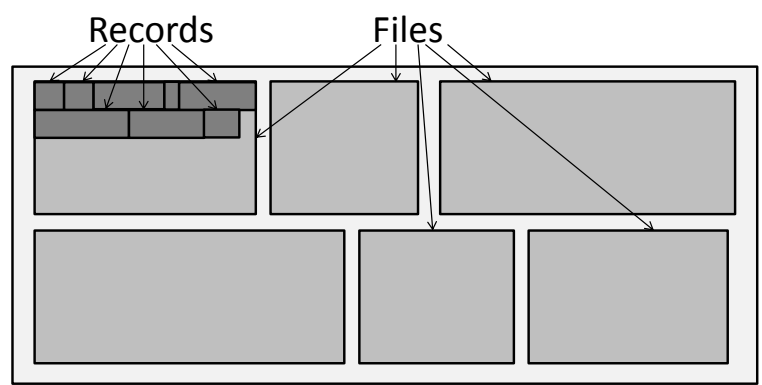

Figure 3: Logical View of File and Record Layout

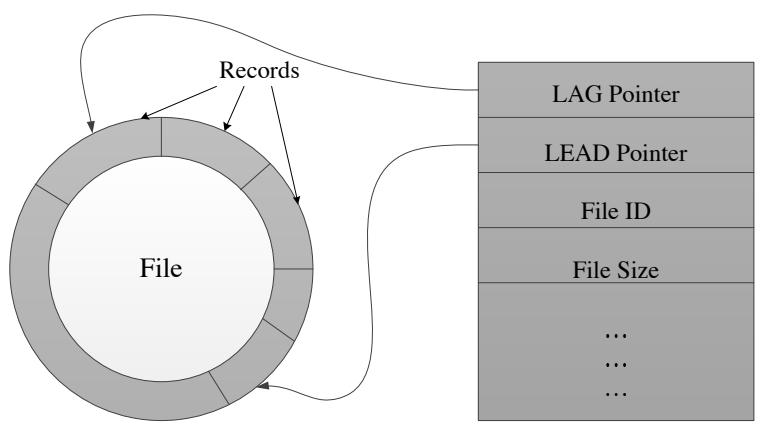

Figure 4: File Metadata in RAM and FRAM

circular storage strategy matches the needs of most sensing applications and provides inherent wear-leveling guarantees. The number of files is predetermined, as are the file sizes, and metadata is updated only in FRAM. By configuring file sizes to consume all available flash, near-uniform write access can be achieved. In the event of heterogeneous access rates, higher-rate files can be allocated more space than lower-rate files to achieve improved wear-leveling.

Files in flash memory are block-aligned to account for flash write and erase constraints. Updates to a file always occur through the write-back cache resident in FRAM. This ensures efficient batching of writes and further reduces flash wear, discussed further in Section 4.4.

\subsection{File Metadata}

Files are represented in primary memory (and in FRAM) as file metadata. LoggerFS uses constant-sized metadata to represent each file; the size does not increase with file size. Since the number of files is preconfigured, the amount of space required to store the file metadata in FRAM is known during installation. This allows LoggerFS to assign space for the write-back cache after accounting for the space required for the associated data structures.

Figure 4 illustrates the metadata associated with each file. A pair of pointers, LAG and LEAD, are used to maintain the circular files. The LAG pointer points to the oldest record, while the LEAD pointer points to the newest record. The size of the record is contained within the record itself, allowing minimal information to be stored in metadata, in addition to supporting variable-sized records. Additional information, such as the file identifier and file size, are stored as part of the file metadata to support fast file access and update.

\subsection{File System Consistency}

A lightweight, consistent data structure is used by LoggerFS to guarantee file system consistency. Every variablesized record is first converted to a sequence of writes, the last of which is an atomic write operation. Consider Figure 5, which illustrates the idea behind the consistent data structure. All updates to a file in LoggerFS occur through FRAM via the write-back cache. Each variable-sized block of data (record) written in FRAM is converted to a pointer update. The pointer contains the starting address of the record and is only updated after the block of data has been successfully written to FRAM. A one-bit flip pointer index is finally updated after the pointer address has been successfully recorded in FRAM.

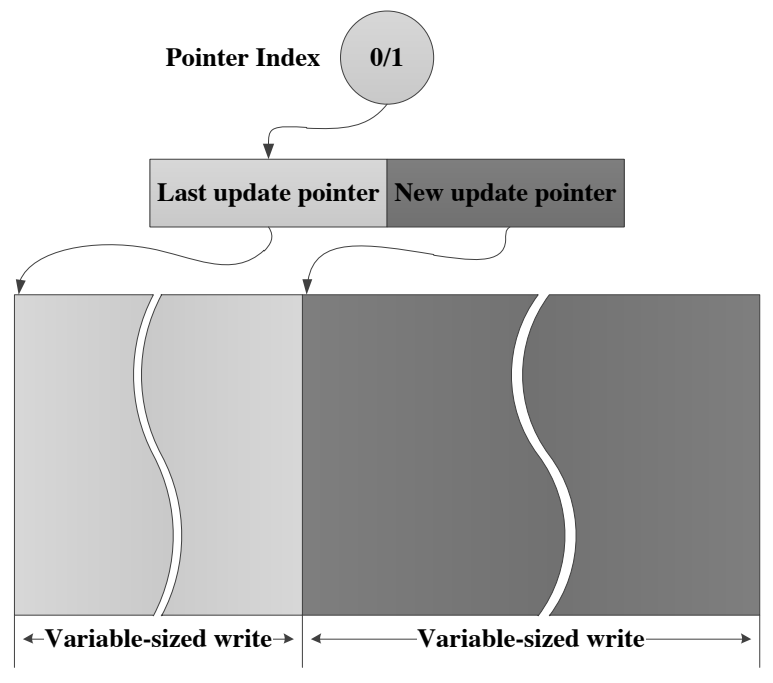

Figure 5: Consistent Data Structure Update

Consider an example. Let the state of the data structure be such that the lightly shaded block in Figure 5 is the last known correctly saved record in FRAM. The lightly shaded last update pointer contains the starting address of this block of data, and the pointer index records a 0 , indicating that the last update pointer is the last known valid value of the pointer. Assume that a new block of data must be written, indicated by the darkly shaded block of data in the figure. After the darkly shaded block of data is written to FRAM, the new update pointer is updated. Following the update, the pointer index is flipped to a 1 . Since the update of a single bit is an atomic operation in any architecture, the entire block update is thus logically converted to an atomic update. A write failure at any point before the pointer index bit update does not corrupt the previous valid record entry.

Reading a record from this structure involves reading the pointer index bit, looking up the corresponding pointer value, and then reading the corresponding block of data using the address stored in the pointer.

Since all data structures used in LoggerFS are consistent, and every write is atomic, we are able to achieve file system consistency without needing any additional software mechanisms, e.g., checkpointing/consistency points.

\subsection{FRAM-based Write-back Cache}

The FRAM-based write-back cache allows LoggerFS to 
handle bursts of high-rate sensor data and alleviates wear on the flash module. The cache is again structured as a circular buffer capable of holding variable-sized files. Caching a file involves moving the file from flash memory to the cache; space for the entire file is allocated in the cache. A pair of LAG and LEAD file pointers are used to point to the least recent and the most recent files which were paged in from flash memory, respectively. The files in turn contain data records maintained by the LAG and LEAD pointers explained in Section 4.2.

Figure 6 presents the read workflow for LoggerFS. File read operations can be served from either cache or flash, depending on where a file is located. If a read operation arrives after a file has been cached for writes or deletes, the read is served from cache. Read operations for an evicted file are served from flash, but the read data (or file) is not cached. This simplifies cache manipulation because all cached files are always dirty and can be flushed to flash when evicted; no additional dirty bits need to be maintained.

Figure 7 presents the write workflow. All file updates occur through the write-back cache. When a record needs to be updated, the existence of the file is first verified in cache. If the file is already cached, the record is updated in cache. Otherwise, the contents of the entire file are first paged into FRAM from flash before the record is updated.

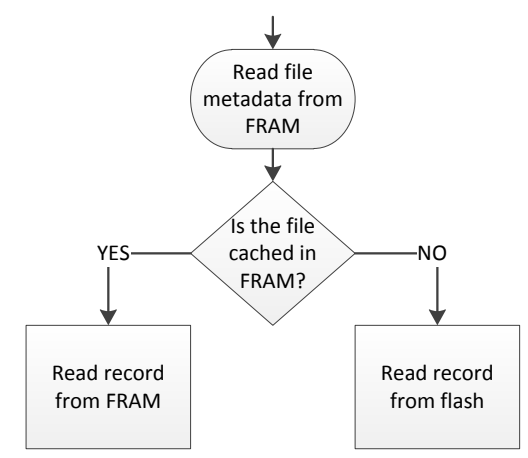

Figure 6: Read Path with Cache

\subsubsection{Cache Eviction}

Cache memory tends to get fragmented over time, often requiring complex data structures to be managed. Since our target devices are resource-constrained, we use a FIFO file eviction policy, which eliminates in-memory cache fragmentation. Since the cache itself is a circular buffer, newly cached files are always written to the end of the buffer. Memory for the new file is allocated immediately after the most recent file entry in FRAM. If this new allocation causes the file to extend beyond the least recent file entry, the least recent file(s) is/are evicted until there is enough space to store the new file. File sizes are therefore limited by FRAM size.

\subsubsection{Cache Data Persistence}

Write operations to FRAM are faster than writes to flash (Table 1), making FRAM a suitable choice for a write cache. Additionally, the size of FRAM is typically much larger than the size of RAM, at a fraction of the cost. Using FRAM for caching enables LoggerFS to buffer large amounts of data during high-rate data bursts. To ensure reliability, records

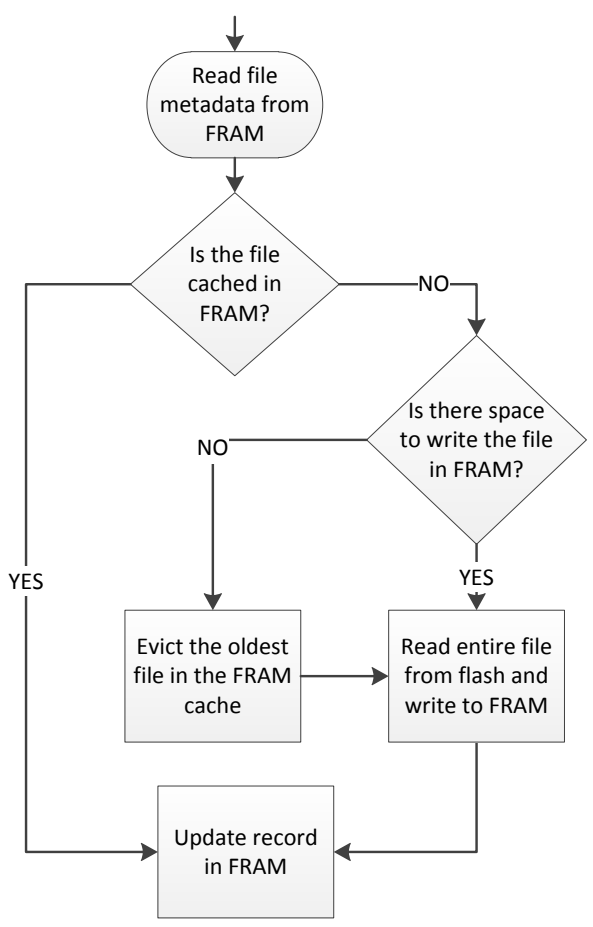

Figure 7: Write Path with Cache

written to cache use the consistent data structure approach described in Section 4.3. As non-volatile memory, FRAM allows all data stored in the cache to be available across device failures without the need for cache rewarming.

\section{FILE SYSTEM IMPLEMENTATION}

We implemented LoggerFS in $\mathrm{C}$ for our custom sensing platform. The MCU, an ATMega644 [2], includes 64KB of in-system-programmable flash memory, $2 \mathrm{~KB}$ of EEPROM, and $4 \mathrm{~KB}$ of SRAM. Two external memory modules, a $64 \mathrm{~KB}$ FRAM [26] and a 512KB flash [1], were added to support the file system. The implementation presented here and all associated experiments were conducted on this resourceconstrained platform, demonstrating the lightweight nature of the design.

\subsection{File, Record, and Cache Implementation}

Files comprise multiple records. Listing 1 shows the representation structure of a record header (record header), a file header (fs_header), and a cache header (cache_header). Record headers are included at the start of every record within a file and contain the size of the corresponding record. They also store a monotonically increasing event identifier (event_id) used to support file system consistency checks, and a checksum (record_crc). The event_id is used to verify continuity of update operations after a system is restarted following a crash and prevents further data corruption in the event of an unrecoverable hardware error.

File headers contain a file identifier (file_id), the size of the file (file_size), and a file system event identifier (fs_event_id) used to support consistency checks. File headers also store a LAG pointer (lag_record_ptr) and a LEAD pointer (lead_record_ptr), which contain logical off- 


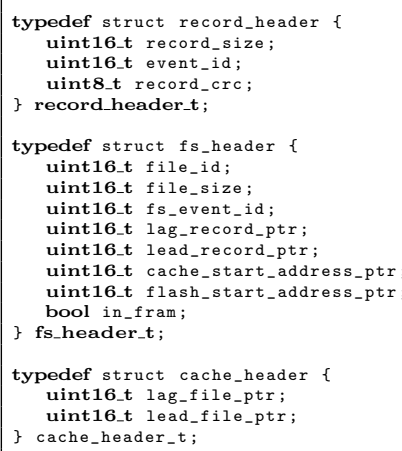

Listing 1: File, Record, and Cache Metadata

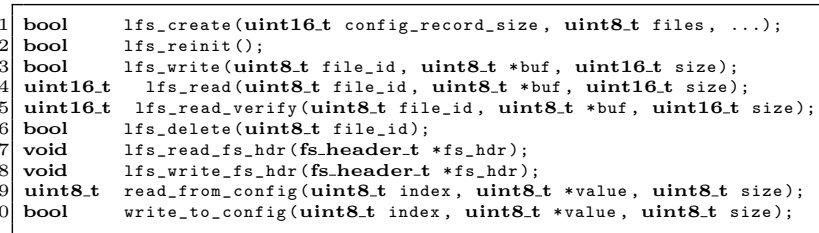

Listing 2: LoggerFS API

set locations. The physical record locations are calculated by adding the logical offsets to the starting address of the file in cache (cache_start_address_ptr), or the starting location of the file in flash memory (flash_start_address_ptr). The starting address in flash is stored as a block address since files are stored block-aligned. The in_fram variable indicates whether a file is present in FRAM cache or in flash memory. Files can simultaneously exist in both FRAM and flash. To accommodate this, the cache_start_address_ptr and flash_start_address_ptr both contain values.

The cache header contains a LAG pointer (lag_file_ptr) and a LEAD pointer (lead_file_ptr) to keep track of contiguous space being used in the circular cache. The pointers point to the oldest and newest cached files, respectively.

\subsection{File System API}

Listing 2 presents the API exposed by LoggerFS. A new file system is created by calling lfs_create(). LoggerFS currently supports a single configuration file which may contain multiple configuration elements. The arguments to lfs_create () consist of the size of the configuration data file (config_record_size), the maximum number of supported files (files), and the size of each file (...), in bytes. A call to lfs_create() initializes FRAM and FLASH. Next, the configuration file size, number of data files, and corresponding file sizes are stored at the start of FRAM. Finally, the file metadata sections for all files are instantiated in FRAM.

The consistent data structure described in Section 4.3 is used to store the file metadata section in FRAM. Two copies of each file metadata entry are maintained; one copy contains the state of the file after the most recent update, while the other copy contains the state of the file before the update. Figure 8 illustrates the use of the consistent data structure to maintain consistent copies of the file metadata.
The new update pointer and last update pointer point to the starting addresses of the new and old metadata entries, respectively. Consistency of record updates is safeguarded by using two copies of LEAD and LAG pointers, respectively stored in two copies of the file metadata.

A magic number is also stored at a fixed FRAM location. During system initialization or restart, lfs_reinit() checks for the existence of a previously installed LoggerFS instance by checking for the presence of this magic number in FRAM. If a file system exists, the file sizes are read from FRAM, and the necessary run-time variables are loaded in to memory. The file and record metadata, which is also stored in FRAM, can then be accessed by the file system.

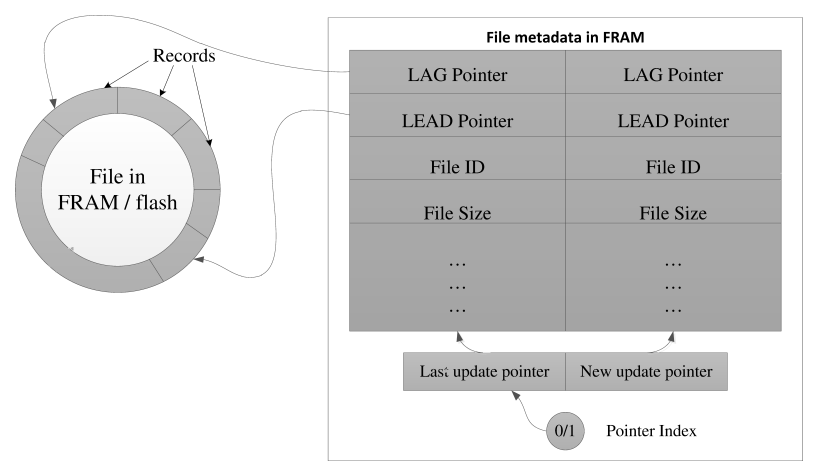

Figure 8: Consistent File Metadata

lfs_write() takes a file identifier (file_id) as argument, the data buffer (buf), and the size of the data buffer (size). A write entails reading the file metadata in $\mathbf{f} \mathbf{s}_{\text {_hdr }} \mathrm{hia}$ a call to lfs_read_fs_hdr() to identify the LAG and LEAD pointers. Using these pointers, the LAG and LEAD records are identified, and the correct write location in FRAM is calculated. Once the record is written, the LAG and LEAD pointers are recalculated and written back as part of the fs_hdr in FRAM via a call to $l \mathbf{f} \mathbf{s}_{-} w r i t e_{-} \mathbf{f} \mathbf{s} h d r()$. The LAG pointer and corresponding record may need to be manipulated since write operations may trigger record deletions if the file is full. The LAG pointer is updated via lfs_increment_lag_pointer () , whereas the LEAD pointer is updated via the lfs_write() function itself, as part of the data write.

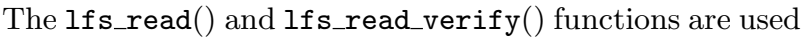
to read the oldest record in a file. Ifs_read_verify() additionally computes a checksum over the read data and compares it to the checksum stored in the record. The checksum verification step makes Ifs_read_verify () slower than Ifs_read(), but useful in applications where data correctness is critical. Sensor applications can choose to save or discard corrupt data. The arguments to the functions include the file identifier (file_id), the read buffer (buf), and the size of the buffer (size). The return value indicates the size of the record read. The lfs_delete () function takes the file identifier ( $\left.f i l e_{-} i d\right)$ as input and deletes the oldest record in the file to create space for new records.

read from config () and write_to_config() are used to handle configuration data. Configuration entries are identified using indices into the configuration file. The configuration data file is not a circular buffer, but is stored using the consistent data structure approach for robustness. 


\section{EVALUATION}

The LoggerFS implementation was evaluated for both reliability and performance. Reliability is measured in terms of the file system's ability to recover from failures while read and write workloads are executing. Performance is characterized in terms of the maximum rate of read and write operations that the file system is able to sustain.

\subsection{Reliability}

The LoggerFS implementation was first validated to ensure its ability to recover from failures. During normal operation, a file system can encounter system failures, such as, power loss due to battery failure, or sub-system failures caused by faulty hardware components. System failures are caused by hardware faults which cause the entire system to go down. Sub-system failures are usually limited to specific hardware components and can be isolated at the software level. We characterize four failure scenarios representative of both types of failures. The four experimental scenarios are enumerated below. To simulate system failures, we introduce power loss during operation (case 1). Sub-system failures are simulated by injecting software faults during write operations to flash, FRAM, or both flash and FRAM simultaneously (cases 2 to 4 , respectively).

1. System failure: During active read and write operations, power was removed from the system, and the system was later checked for consistency after restart.

2. Sub-system flash failure: Writes to flash were made to fail at random intervals (by returning failure codes) during write operations, and the system was later checked for consistency.

3. Sub-system FRAM failure: Writes to FRAM were made to fail at random intervals during write operations to FRAM, and the system was later checked for consistency.

4. Sub-system flash and FRAM failure: Writes to either flash or FRAM were made to fail at random intervals during write operations, and the system was later checked for consistency.

Four files, of sizes $1 \mathrm{~KB}, 2 \mathrm{~KB}, 4 \mathrm{~KB}$, and $8 \mathrm{~KB}$ were created, and a total of 10 trials were run for each case. A 50\%/50\% mixed read/write workload was issued for case 1 , while a $100 \%$ write workload was issued for cases 2,3 , and 4 .

\subsubsection{Results}

For case 1, we non-deterministically remove power to the platform during 1-minute long mixed read and write workloads. For each test, LoggerFS was able to handle system failures gracefully and re-initialize after power loss. The lfs_reinit() function was able to verify a consistent version of the file system installed in both FRAM and flash, and then continue normal operation.

For cases 2, 3, and 4, we ran individual trials for a duration of 1 minute. All the tests involved sending write operations to the four files. For case 2, faults were injected at random intervals during write operations to flash. For case 3 , faults were injected randomly during writes to FRAM. For case 4, faults were injected at random intervals during writes to both flash and FRAM. After 1 minute, all data was read back and verified. All read and verify operations completed successfully. Further, on the next system reboot, lfs_reinit() was able to verify a consistent file system installed on FRAM and flash.

\subsection{Performance}

An embedded sensor can experience brief periods of time when it issues $100 \%$ writes due to sensing operations. Similarly, there are periods of time when the device issues $100 \%$ reads associated with the transmission of data to a base station. There are also brief periods of time when mixed workloads might be observed, where a sensing operation is initiated while data transmission is in progress. To evaluate system performance, we first characterized typical I/O workloads and measured system performance at $100 \%$ write, $100 \%$ read, and mixed read and write workloads. For simplicity of measurement and analysis of mixed workloads, we assumed a $50 \% / 50 \%$ mix of read and write operations. All read and write operations are 256 bytes.

\subsubsection{Hardware Benchmarking}

We first benchmarked the read and write speeds for the FRAM and flash devices used in the LoggerFS implementation. The results allowed us to better characterize expected read and write performance.

Table 2: Measured Read and Write Speeds

\begin{tabular}{|c|c|c|c|}
\hline Device & Reads & Writes & Erase and Write \\
\hline \hline FRAM & $0.8 \mathrm{~ms}$ & $0.6 \mathrm{~ms}$ & -NA- \\
\hline Flash & $1.2 \mathrm{~ms}$ & $1.1 \mathrm{~ms}$ & $4.3 \mathrm{~ms}$ \\
\hline
\end{tabular}

Table 2 presents the measured read and write speeds for the FRAM and flash memory devices. We measured the time required to transfer 1 million buffers with 256 bytes of data each, and then computed the average time required to transfer a 256-byte buffer, for both read and write operations. Read operations from flash are $50 \%$ slower than reads from FRAM; reading 256 bytes from flash takes 1.2 $m s$, while it requires $0.8 \mathrm{~ms}$ to read from FRAM. Writing 256 bytes of data from flash takes $1.1 \mathrm{~ms}$, which is $83.34 \%$ slower than FRAM, which requires $0.6 \mathrm{~ms}$. However, when the time required to erase a block is amortized across write times, flash is about $600 \%$ slower, at $4.3 \mathrm{~ms}$. This write speed advantage allows FRAM to handle bursts of high-rate data up to $426.67 \mathrm{KBps}$, and to sustain write throughputs up to 7 times faster than flash memory, at $59.53 \mathrm{KBps}$.

\subsubsection{Write I/O Performance}

We measured the write performance of LoggerFS assuming variable degrees of cache availability, including no cache. We considered a $100 \%$ write workload and simulated conditions where a percentage of writes cause a cache miss. A $0 \%$ cache hit rate represents the case where every new record update causes file eviction(s) from FRAM and a read from flash; whereas a $100 \%$ cache hit rate does not cause a file eviction. We create a total of 1,000 files of size 256 bytes each, containing one record each. This allows us to model the amount of data evicted from FRAM and subsequently read in from flash. We know that the size of the evicted file is the same as the size of the file which is being paged in.

Figure 9 summarizes the write throughput observed at variable cache hit rates. The $\mathrm{x}$-axis represents the write cache hit rate, and the $y$-axis represents the throughput 


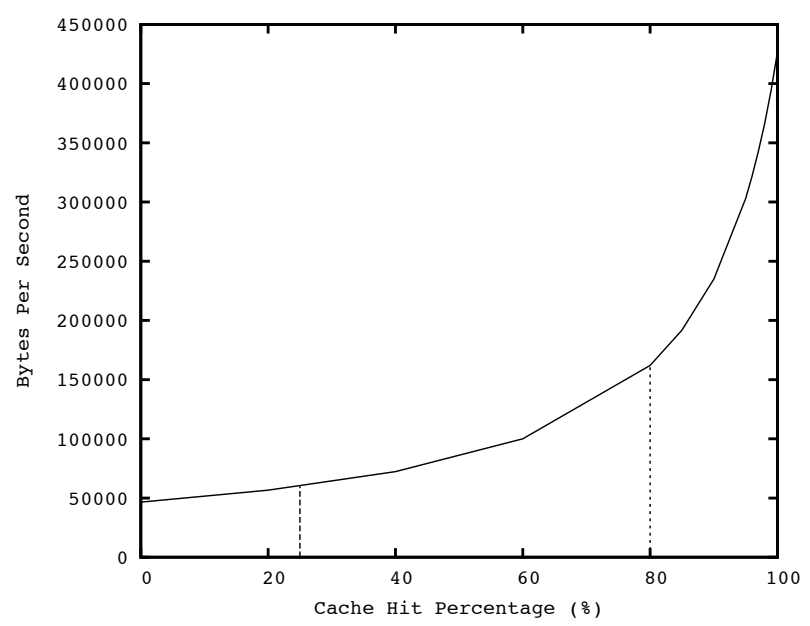

Figure 9: Write Throughput with Cache

achieved. In the absence of the write cache, LoggerFS is able to achieve write throughput of approximately $59.53 \mathrm{KBps}$. In the presence of a write cache and a $0 \%$ hit rate, LoggerFS is only able to achieve write throughput of approximately $46.55 \mathrm{KBps}$. This can be attributed to the additional overhead associated with file evictions.

With the write cache implementation and a $25 \%$ hit rate, LoggerFS achieves write throughput parity with the cachefree implementation. At a cache hit rate of approximately $80 \%$, we observe throughput of $162.02 \mathrm{KBps}$. Beyond $80 \%$, we observe almost exponential growth in throughput. At $100 \%$, when LoggerFS is able to utilize pure FRAM update speeds, a maximum throughout of $426.67 \mathrm{KBps}$ is observed, which is about 8 times faster than the LoggerFS implementation without cache.

\subsubsection{Read I/O Performance}

We next measured the read performance of LoggerFS with varying cache availability. It is easier to simulate read cache hit rates (rather than writes), as we do not need to account for evictions. A $0 \%$ read cache hit rate is created by reading a file which is entirely stored in flash, while a $100 \%$ hit rate results in reading all data from FRAM.

Figure 10 summarizes the throughput achieved at varying cache hit rates. The $\mathrm{x}$-axis represents the read cache hit rate, and the y-axis represents the throughput achieved. In the absence of a cache, LoggerFS is able to achieve read throughput of about $213.34 \mathrm{KBps}$. At a $100 \%$ cache hit rate, LoggerFS is able to achieve a read throughput of approximately $320 \mathrm{KBps}$, which is about $50 \%$ faster than the throughput achieved without cache. The difference in throughput between $0 \%$ and $100 \%$ cache hit rates is not large, since flash and FRAM read speeds are similar orders of magnitude.

\subsubsection{Mixed I/O Performance}

Figure 11 summarizes the performance for a 50\%/50\% $\mathrm{read} /$ write workload. The read and write operations in the workload were interleaved. We ran experiments by varying the write cache hit rate from $0 \%$ to $100 \%$, in increments of $20 \%$. For each write cache hit rate increment, the read cache hit rates were simultaneously varied from $0 \%$ to $100 \%$, in increments of $20 \%$. The $\mathrm{x}$-axis represents the read cache hit rate, and the $y$-axis represents the throughput achieved. The

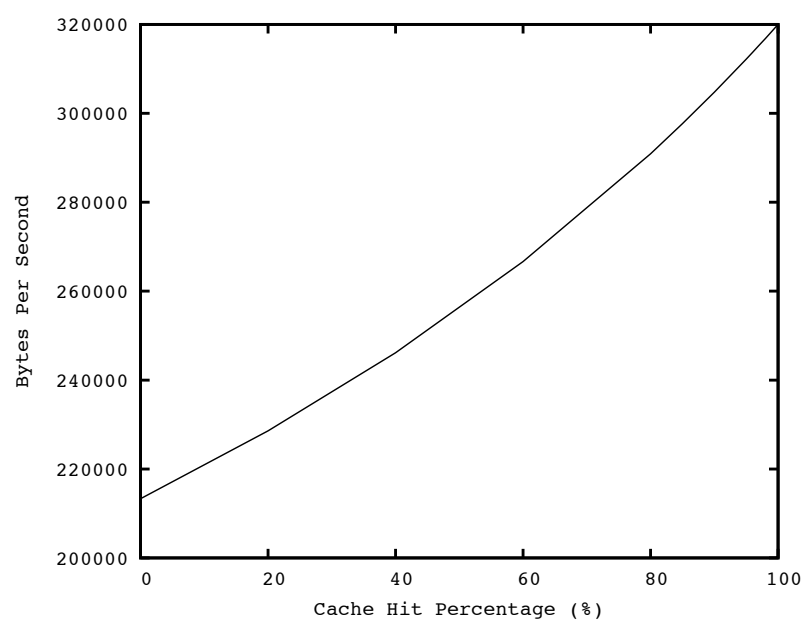

Figure 10: Read Throughput with Cache

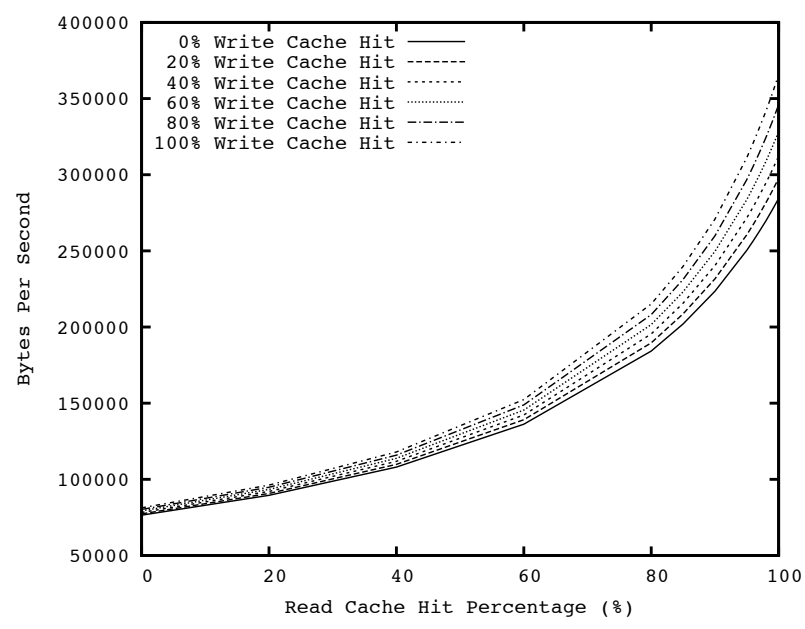

Figure 11: 50\%/50\% Read-Write Throughput

series represent the various write cache hit rates considered. For example, the solid line charts throughput when the write cache hit rate was set at $0 \%$, and the read cache hit rates were changed. The total throughput includes both reads and writes.

Due to the single-threaded nature of LoggerFS, read and write operations are serviced sequentially, and throughput is latency sensitive. We observed that the throughput (at any cache hit rate) can be calculated from the throughput of individual read and write operations from Figures 9 and 10. The observed throughput at $0 \%$ is approximately $76 \mathrm{KBps}$, while the observed throughput at $100 \%$ is approximately 5 times faster, at $365 \mathrm{KBps}$. The observed data transfer rates track the lower of the read and write throughputs. At lower cache hit percentages, throughput is dominated by write speeds, while at over $80 \%$, the read speeds dominate.

Overall, these results demonstrate that the LoggerFS implementation with the FRAM write-back cache performs about 8 times faster than without the cache for write workloads. For read workloads, the FRAM cache helps boost throughput by $50 \%$. For $50 \%-50 \%$ mixed read and write workloads, LoggerFS with the FRAM write-back cache is able to achieve speeds 5 times faster than the implementation without the cache. 


\section{CONCLUSION}

We presented the design and implementation of LoggerFS, a fast, lightweight, and reliable file system. LoggerFS uses a hybrid memory model comprising RAM, FRAM, and flash memory. The file system is designed to use fixed-sized metadata, resulting in a small main memory footprint. Metadata is stored in FRAM for fast reads and writes. Data persistence and reliability are achieved using a consistent data structure update mechanism, ensuring that all updates in the file system are atomic.

We also presented a FRAM-based write-back cache design for LoggerFS. The write-back cache allows LoggerFS to handle bursts of high-rate writes. Data is evicted from cache to flash lazily, when the cache is completely full. We demonstrated fault tolerance features of LoggerFS in the presence of complete system and sub-system failures. We also characterized read and write performance and demonstrated between $50 \%$ and $800 \%$ speed-ups for various workloads using the FRAM write-back cache.

\section{ACKNOWLEDGMENT}

This work was supported by the NSF grants CNS-1545705 and CNS-1541917.

\section{REFERENCES}

[1] Atmel. 4Mb SPI serial flash memory. http://media.digikey.com/pdf/Data Sheets/Atmel PDFs/AT25DF041A.pdf.

[2] AVR. ATmega datasheet. http://www.atmel.com/dyn /resources/prod_documents/doc2593.pdf, 2010.

[3] Qing Cao et al. The LiteOS operating system: Towards unix-like abstractions for wireless sensor networks. In Proceedings of IPSN'08, pages 233-244, Washington, DC, USA, 2008. IEEE Computer Society.

[4] Aleph One Company. YAFFS (Yet Another Flash File System). http://www.aleph1.co.uk/yaffs/yaffs.html/.

[5] Rone Ilídio da Silva et al. Spatial query processing in wireless sensor network for disaster management. In Proceedings of WD'09, pages 194-198, 2009.

[6] Hui Dai et al. ELF: An efficient log-structured flash file system for micro sensor nodes. In Proceedings of SenSys '04, pages 176-187, New York, NY, USA, 2004. ACM.

[7] In Hwan Doh et al. Exploiting non-volatile RAM to enhance flash file system performance. In Proceedings of EMSOFT'07, pages 164-173, 2007.

[8] Cypress Semiconductor Doug Mitchell. nvSRAMs eclipse battery-backed memory. http://www.cypress.com/?docID $=9279$.

[9] Adam Dunkels. Programming Memory-Constrained Networked Embedded Systems. PhD thesis, SICS, 2007.

[10] Adam Dunkels, Bjorn Gronvall, and Thiemo Voigt. Contiki - A lightweight and flexible operating system for tiny networked sensors. In Proceedings of $L C N^{\prime} 04$, pages 455-462, 2004.

[11] Eran Gal and Sivan Toledo. A transactional flash file system for microcontrollers, 2005.

[12] David Gay. Matchbox: A simple filing system for motes. http://www.docs.tinyos.net/tinyos1.x/doc/matchbox.pdf.

[13] Jonathan W. Hui and David Culler. The dynamic behavior of a data dissemination protocol for network programming at scale. In SenSys'04, pages 81-94. ACM Press, 2004.

[14] Texas Instruments. MSP430 Ultra-low-power microcontrollers. http://www.ti.com/lit/sg/slab034w/slab034w.pdf.

[15] Jaein Jeong. Node-level representation and system support for network programming, 2003.

[16] Jaein Jeong and David Culler. Incremental network programming for wireless sensors. In $S E C O N^{\prime} 04$, pages 25-33, 2004.

[17] Jaein Jeong et al. Network reprogramming. TinyOS document, http://webs.cs.berkeley.edu/tos/tinyos1.x/doc/NetworkReprogramming.pdf.

[18] Eun-ki Kim et al. FRASH: Hierarchical file system for FRAM and flash. In Proceedings of ICCSA'O', pages 238-251, 2007.

[19] Sukun Kim et al. Wireless sensor networks for structural health monitoring. In Proceedings of SenSys '06, pages 427-428, New York, NY, USA, 2006. ACM.

[20] Philip Levis, Sam Madden, Joseph Polastre, Robert Szewczyk, Alec Woo, David Gay, Jason Hill, Matt Welsh, Eric Brewer, and David Culler. TinyOS: An operating system for sensor networks. In Ambient Intelligence. Springer Verlag, 2004.

[21] Biswajit Mazumder and Jason O. Hallstrom. An efficient code update solution for wireless sensor network reprogramming. In Proceedings of EMSOFT '13, New York, NY, USA, 2013. ACM.

[22] Joseph Polastre, Robert Szewczyk, and David Culler. Telos: Enabling ultra-low power wireless research. In Proceedings of IPSN '05, 2005.

[23] Mendel Rosenblum and John K. Ousterhout. The design and implementation of a log-structured file system. ACM Transactions on Computer Systems, 10:1-15, 1992.

[24] Cypress Semiconductor. CY14B104N datasheet. http://www.cypress.com/?rID=39015.

[25] Cypress Semiconductor. FM22L16 4Mb asynchronous F-RAM memory. http://www.cypress.com/?docID $=42532$.

[26] Cypress Semiconductor. FM25V05 512Kb serial FRAM. http://www.cypress.com/?docID $=47930$.

[27] Livio B Soares et al. Metadata snapshotting: A simple mechanism for file system consistency, 2003.

[28] Crossbow Technology. Mote in network programming user reference. TinyOS document, http://webs.cs.berkeley.edu/tos/tinyos1.x/doc/Xnp.pdf.

[29] Nicolas Tsiftes et al. Enabling large-scale storage in sensor networks with the Coffee file system. In Proceedings of IPSN'09, pages 349-360, Washington, DC, USA, 2009. IEEE Computer Society.

[30] Geoff Werner-Allen et al. Fidelity and yield in a volcano monitoring sensor network. In Proceedings of OSDI '06, pages 381-396, Berkeley, CA, USA, 2006. USENIX Association.

[31] David Woodhouse. JFFS : The Journalling Flash File System, 2001.

[32] Jin Hyuk Yoon et al. Chameleon: A high performance flash/FRAM hybrid solid state disk architecture. IEEE Computer Architecture Letters, 7(1):17-20, 2008. 